\title{
El Semillero en Investigación Formativa en Historia, Cultura Política y Educación y sus experiencias alternativas para la investigación social $\epsilon$ histórica
}

\author{
Anderson Paúl Gil Pérez* \\ Christian Camilo Calderón Gil**
}

\section{Introducción}

El Semillero en Investigación Formativa en Historia, Cultura Política y Educación, es una comunidad de aprendizaje conformada por estudiantes de las licenciaturas en Etnoeducación y Desarrollo Comunitario y Español y Literatura, pertenecientes a la Facultad de Ciencias de la Educación de la Universidad Tecnológica de Pereira (UTP). que desde hace aproximadamente cinco años se viene preocupando por observar e indagar los temas relacionados con la identidad universitaria, construcción de ciudadanía y

* $\quad$ Estudiante Licenciatura en Etnoeducación y Desarrollo Comunitario. Quinto semestre. Universidad Tecnológica de Pereira.pauljuncogp@gmail.com, apgil@utp.edu.co

** Estudiante Licenciatura en Español y Literatura. Noveno semestre. Universidad Tecnológica de Pereira. christian7@utp.edu.co,camilo7768@gmail.com

ITINERARIO EDUCATIVO • AÑO XXVI, N. ${ }^{\circ} 59$ • I99-ZI4 • ENERO - JUNIO DE ZOIL 
recuperación de la memoria histórica de la Universidad y de la ciudad de Pereira. Quiere decir que el semillero se pregunta constantemente por los aspectos, problemas y acontecimientos que se pueden encontrar en la relación ciudad-universidad.

Adicionalmente, el semillero pertenece al Grupo Políticas, Sociabilidades y Representaciones Histórico Educativas, categoría B Colciencias, con el cual se ha logrado desarrollar un trabajo colaborativo de constante asesoramiento y discusión en torno a los intereses académicos. También se ha preocupado por participar de procesos de cooperación institucional; por ejemplo, participó junto con la Misión de Observación Electoral (MOE), en el seguimiento a medios de comunicación y veeduría en los procesos electorales de 2010 y 2011; los aprendizajes político-electorales obtenidos nos han servido mucho para ampliar la mirada sobre algunas de las investigaciones en curso.

La dinámica de trabajo que se realiza en el semillero, constituye una apuesta tradicional y al mismo tiempo alternativa, tradicional en el sentido que se conjugan elementos del trabajo cotidiano de los semilleros, es decir, reuniones periódicas para discutir algunas lecturas; y alternativa porque las actividades que se desarrollan implican mayor explicación e intercambio de saberes, además un ejercicio de comunicación emergente que posibilita más autorreflexividad sobre las fuentes, el trabajo con autores, la elaboración de proyectos que puedan competir por financiación.

Como su nombre lo indica este semillero se orienta bajo los planteamientos de la investigación formativa diferenciándose de la formación investigativa. Por ello seguimos la tesis propuesta por el profesor Bernardo Restrepo Gómez, donde señala que:

La investigación formativa es formar en investigación y para la investigación, desde actividades investigativas que incorporan la lógica de la investigación y aplican métodos de investigación 
(...) [en cambio la] Formación investigativa es formar para la investigación desde otras actividades no propiamente investigativas, como cursos de investigación, lectura y discusión de informes de investigación, etc. (2004: 3).

En este sentido, sin negar la importancia de la instrucción pedagógica que procura desarrollar en el sujeto la competencia investigativa, queremos resaltar la aplicación de la investigación formativa en el semillero, promoviendo valores, actitudes y aptitudes investigativas, capacidad de trabajo en equipo, liderazgo, distribución y cumplimiento de responsabilidades. En resumidas cuentas es una apuesta a pensar la investigación como un quehacer práctico y no solamente como un fin. Este giro en la mirada planteado por María Teresa Uribe de Hincapié, en el prólogo del libro Estrategias de investigación social cualitativas de María Eumelia Galeano Marín, consiste en:

Situar la mirada en el sujeto de la acción, (...) en sus diferencias y las distintas maneras de vivir y pensar (...) retornar al primer plano del escenario de la investigación donde los sujetos, individuales o colectivos, con sus prácticas sociales, sus memorias y sus olvidos, sus propósitos de cambio, resistencia o sometimiento (...) [exigen] interpretar, clasificar, comparar y enunciar las situaciones o procesos observados y para desentrañar las lógicas y maneras diferenciadas de vivir en sociedad (2004:11).

En vista a lo anterior hemos considerado que los aspectos que profundizaremos durante esta ponencia son alternativos -y merecen ser presentados en este coloquio sobre investigación de métodos alternativos en las ciencias sociales-porque la investigación no es un proceso autómata y más bien es autorreflexivo y requiere de una observación constante, de la implementación de nuevas técnicas y la transformación de otras que parecieran agotadas, pero que debido a miradas amplias con tintes creativos surgen y producen

ITINERARIO EDUCATIVO • AÑO XXVI, N. ${ }^{\circ} 59$ • I99-ZI4 • ENERO - JUNIO DE ZOIL 
nuevas o distintas miradas sobre lo social aunque implican la apropiación de la lógica del proceso investigativo.

Ahora vamos a compartirles algunas experiencias investigativas que nos han permitido aprender a sortear obstáculos a través de métodos y herramientas -adquiridas en el semillero que posibilitan plantear y responder problemas- utilizados en la realización de dos proyectos en torno a la celebración de los 50 años de la Universidad Tecnológica de Pereira, con el fin de rescatar la memoria -oral y escrita- inmersa en los eventos significativos de la historia de la universidad.

\section{«Memorias Universitarias UTP 50 años marcando la historia de la región»}

Memorias Universitarias UTP. 50 años marcando la historia de la región surgió a raíz de la necesidad de nuevas preguntas sobre los procesos que no han sido contados o han sido invisibilizados en la historia institucional, partiendo precisamente de fuentes institucionales como el Archivo Histórico de la Universidad Tecnológica de Pereira y la Hemeroteca de la biblioteca pública Ramón Correa Mejía de Pereira, y sin dejar de tomar en cuenta la memoria de las personas que trasegaron desde la creación de la UTP hasta el presente. Es decir, el desencuentro con temas que se refieren a la formación orgánica y a los personajes honorarios como rectores; y el encuentro con otros temas mucho más sociales. (Delgado, 2011) y (Gil, 2011).

Durante el desarrollo de esta investigación fueron muchos los aprendizajes, porque para nosotros era la primera vez que nos acercábamos a las dinámicas de un semillero y a una investigación. Por ejemplo, no sabíamos en qué consistía el trabajo con las fuentes, cómo se hacen fichas bibliográficas; distinciones elementales que para los investigadores son tan básicas, cómo hablar de fuentes primarias y secundarias. Es innegable que en 
un inicio asumimos la investigación y el trabajo de campo en el semillero desde miradas superficiales en donde solo atendíamos a la literalidad de las fuentes sin subrayar las relaciones que existen entre ellas. No obstante, a medida que ahondábamos en la valiosa información registrada en actas, periódicos y entrevistas pudimos conectar las distintas maneras de narrar los sucesos hasta el punto de llegar a comprender la diversidad de relaciones. Podemos afirmar que nuestra observación sobre los procesos fue mucho más profunda y reflexiva. Acercándonos a lo propuesto por Jesús Galindo (1998): «la mirada que mira a la mirada que mira».

Varias preocupaciones, limitaciones y problemas se debieron afrontar en este proyecto, una de ellos fue la experiencia de manejar categorías de investigación, en nuestro caso teníamos inicialmente varias por abordar: protesta social, movimiento social, acción colectiva y organización social. Autores como: Sídney Tarrow y Charles Tilly, Mauricio Archila, Álvaro Acevedo Tarazona y María Teresa Uribe, nos dieron luces sobre algunas de nuestras inquietudes. Nos reunimos para determinar cuáles eran las categorías más apropiadas que nos permitieran privilegiar lo inductivo sobre lo deductivo.

Al reunirnos nos quedó en claro que cuando se empieza en la investigación es necesario mantener un equilibrio entre el riesgo y la prudencia, apostándole a propuestas más descriptivas eludiendo exageradas ambiciones analíticas. Para nuestro caso y después del asesoramiento y discusión con el tutor, determinamos que el repertorio de la protesta social asimilado a una categoría servía mucho más para cumplir el propósito de reflejar lo que deseábamos mostrar acerca de los cincuenta años de la Universidad.

Otro de los proyectos desarrollados por el semillero y que evidencia el uso de las metodologías propias de la investigación formativa es:

ITINERARIO EDUCATIVO • AÑO XXVI, N. ${ }^{\circ} 59$ • I99-ZI4 • ENERO - JUNIO DE ZOIL 


\section{Memorias que no se jubilan: un acercamiento a las historias de vida de los jubilados de la UTP}

Es un proyecto de investigación que apenas se encuentra en sus primeras fases de ejecución, por ello, nos interesa resaltar su construcción como propuesta, tanto así que obtuvo el segundo puesto en la convocatoria de proyectos de extensión organizada por la Vicerrectoría de Investigación, Innovación y Extensión en la UTP en el segundo semestre de 2011.

La investigación plantea a veces algunas contradicciones o ambigüedades en quienes la practican, no se sabe qué momento presenta mayores retos, si su estructuración o su desarrollo. Desde la concepción de la idea de investigación hasta la ejecución de la misma aparecen nuevos elementos -perspectivas no contempladas en la planeación inicial a pesar de que en ella siempre se intenta conocer todas las posibilidades- que requieren ser abordados o por lo menos tomados en cuenta para próximos problemas investigativos.

Como lo señalamos anteriormente, el primer proyecto Memorias UTP 50 años Marcando la Historia de la Región, en una de sus fases, comprendió la realización de entrevistas a profesores jubilados y estudiantes de la UTP, donde descubrimos el potencial histórico contenido en la memoria de estas personas, testimonios sobre momentos fundamentales en la construcción de la Universidad, contradicciones por rescatar que a nuestro modo de ver representan una disyuntiva entre la Universidad institucional y la Universidad humana. Observamos un problema a investigar al que tuvimos que darle forma mediante una propuesta viable y atractiva para la Universidad.

Para ello debimos hacer un primer rastreo teórico, a manera de balance bibliográfico sobre otros autores como Maurice Halbwachs, Paul Ricoeur, y Paul Thompson, tratando de encontrar desde ellos maneras de plantear, de darle sustento teórico a nuestra idea. 
Comprender las propuestas epistemológicas de un autor, requiere leerlo en varias ocasiones, conocer algún contexto sobre lo que fue el autor y al menos tener claridad de a qué época perteneció para a partir de allí entender los dilemas que como autor debió o quiso responder con su obra. Así tratamos de realizarlo nosotros desde el semillero y de los resultados de este intento por trabajar con las ideas de estos hombres surgieron aprendizajes que para un coloquio como este son importantes señalar, porque recuerdan la manera como se empieza a construir un camino propicio para la investigación.

En ese intento nuestro por apropiarnos de aquellos temas, nos perdimos en un sinnúmero de conceptos, que produjo que trabajáramos algunas veces sobre ideas y supuestos equivocados, dando tumbos, palos de ciego, momentos en los cuales fue vital la guía del tutor para continuar por «el buen camino».

La experiencia de perderse en medio de la teoría, nos ayudó a entender que el proceso de la investigación es una construcción paso a paso, muy estricta, que se regula por principios sistémicos y organizativos a fin de evitar las confusiones conceptuales. La aptitud investigativa va más allá de los cursos meteorológicos e incluye la práctica investigativa, que denominamos investigación formativa.

Hemos tratado de mostrar algunos de los aprendizajes que cada investigación nos proporcionó. Son muchos más, pero estamos interesados en expresarles un relato de autoaprendizaje, que evidencie las características del semillero, sus prácticas de investigación formativa y los conocimientos que hemos logrado adquirir sobre métodos, técnicas, medios, habilidades y herramientas para asumir la investigación, como una búsqueda de rigor académico siempre presente. Ahora vamos a comentarles cómo trabajamos desde el semillero, cada una de estas herramientas y técnicas que la investigación implica. No todas las herramientas han sido uti-

ITINERARIO EDUCATIVO • AÑO XXVI, N. ${ }^{\circ} 59$ • I99-ZI4 • ENERO - JUNIO DE ZOIS 
lizadas en las dos investigaciones pero sí en la dinámica general del semillero.

\section{Escogencia y trabajo del corpus bibliográfico}

Es una de las fases más importantes de la investigación en donde siempre hemos estado acompañados por el tutor ${ }^{1}$ y su constante invitación al rigor y creatividad quien inicialmente direcciona la ardua tarea de conectar ideas en torno a la bibliografía pertinente, de acuerdo con su experiencia y mirada otrora sobre los problemas investigativos. Así pues, lo primero es aprender a leer, reseñar y debatir los textos señalados por el tutor, actividad encaminada a desarrollar el espíritu indagador. La bibliografía recomendada por el tutor no constituye camisa de fuerza pero sí, debe ser asumida con responsabilidad con el objetivo de propiciar la búsqueda constante de nueva bibliografía.

Por esto, la búsqueda bibliográfica fue un momento colaborativo conducido bajo una pedagogía constructivista que propició que nosotros propusiéramos estudiar otros autores distintos a los recomendados por el asesor: en estos casos se realizan lecturas colectivas para debatirlos y finalmente descartarlos o integrarlos al corpus bibliográfico.

\section{Referencias bibliográficas}

Es normal -y muy lamentable- que los estudiantes en programas como los nuestros donde el componente investigativo es reducido, aprueben algunas materias sin practicar el ejercicio concerniente

1 Nos parece importante señalar que nuestro tutor es John Jaime Correa Ramírez, Historiador de la Universidad Nacional, Magister en Ciencia Política de la Universidad de Antioquia, y doctorando en Ciencias de la Educación - Rudecolombia. Actualmente profesor de tiempo completo en la Universidad Tecnológica de Pereira, Facultad de Ciencias de la Educación, Escuela de Ciencias Sociales. jjcorrea@utp.edu.co 
a informes de lecturas o el fichaje bibliográfico. Por consiguiente darle efectiva ejecución a la fase bibliográfica en las dos investigaciones nos obligó a tomar en cuenta «manuales metodológicos» que explican los puntos que se deben privilegiar, tales como: los argumentos del autor y cómo nombrarlos en nuestros textos a través de un adecuado manejo de las normas de citación APA.

Como investigadores en formación debimos enfrentarnos a esta falencia, donde fue fundamental la cooperación entre el grupo de investigación y el semillero. En el grupo de investigación la mayoría de sus integrantes ya están realizando maestrías y doctorados, contando con óptimas maneras de reseñar y son conscientes de la importancia que tiene este paso para el investigador que se forma. Es muy rescatable para esta ponencia de tipo autorreflexiva resaltar el apoyo brindado por los investigadores formados hacia nosotros los investigadores en formación.

\section{Las huellas del pasado: fichar las fuentes}

Para empezar la indagación en el Archivo Histórico de la Universidad Tecnológica de Pereira, debíamos entender el procedimiento de fichaje de las Actas del Consejo Directivo, Superior y Académico de la institución, para posteriormente poder trabajar la información y consolidar los resultados. Esto significó superar la limitación de un primer acercamiento al trabajo en archivo entendido como la simple toma desmedida de apuntes que se consignan en el trajinado cuaderno de las demás materias y asimilarlo como uno de los momentos fundamentales de la investigación, por ser la primera vez que nosotros nos relacionábamos con la que en adelante sería nuestra materia prima: estantes llenos de folios, expedientes y anexos consignados en hojas amarillentas, únicas huellas del pasado que hoy vemos como reliquias conservadas de años venturosos que constituyen nuestra historia. Además, es así mismo, la puerta de entrada a un universo polifónico de voces escritas, donde surgen algunos de los hallazgos más importantes de procesos como el que venimos realizando.

ITINERARIO EDUCATIVO • AÑO XXVI, N. ${ }^{\circ} 59$ • I99-ZI4 • ENERO - JUNIO DE ZOIS 
Aprender a buscar aquellas huellas del pasado constituye desde nuestra perspectiva una característica que no garantiza ser investigador, pero sin la cual el proceso de formación ni siquiera podría haber empezado. Era obligatorio entonces manejar aspectos puntuales como: apuntar la fecha del documento reseñado, dejar en claro su denominación, escribir el ente productor, señalar los principales actores que intervienen y cómo lo hacen, plantear observaciones y pendientes que se deban cotejar con las demás fuentes, por ejemplo, la hemeroteca; y seriar las fichas evitando que se repitan. Los anteriores son requerimientos básicos que se encuentran en una guía sobre historiografía, pero desde el ejercicio investigativo se interiorizan más adecuadamente.

Por último, es claro que la agilidad y efectividad para realizar estas actividades de fichaje, no constituyen un aprendizaje rutinario como saber montar bicicleta, requiere, más bien, de la constante práctica. A veces es necesario incluso volver al archivo después de haber construido el informe para constatar datos.

\section{Planeación, realización y transcripción de entrevistas}

La escogencia de las personas a entrevistar se hizo, según lo señalaban los primeros datos obtenidos en el archivo. Incluso pareciese que el archivo histórico tomara vida y se convirtiera en un ente que nos muestra uno a uno quiénes son las personas que se deben entrevistar.

Su realización presentó diferentes inconvenientes a superar de acuerdo al entrevistado. Es equívoco suponer que las personas siempre están dispuestas a ser entrevistadas; por muchos factores no siempre es así. En algunas ocasiones aunque se muestran interesadas, tienen poca disponibilidad de tiempo; en otras, se atemorizan ante la responsabilidad de sus propios testimonios. 
Tras la concertación el problema recayó en cómo lograr que nuestros intereses no se sobrepusieran al ambiente de tranquilidad e intimidad que requieren las entrevistas, el fin era evitar la mirada utilitarista de la entrevista señalada por el profesor canadiense Joselyn Létourneau, en su libro la Caja de herramientas del joven investigador (2007:169) «la persona entrevistada puede ser considerada en su papel de actor social o bien puede ser objetivizada como sujeto-testigo, [pero] no puede ser un objeto-testigo».

Pero sin lugar a dudas, es seguro que por más tiempo que se invierta en la planeación de las entrevistas, siempre habrá un margen de incertidumbre que puede ofrecer miradas sobre el entrevistado que no teníamos contempladas y que sería lamentable perderse.

$\mathrm{Al}$ efectuar las transcripciones cometimos la "primiparada» de transcribir la totalidad de las entrevistas, ahora sabemos que lo pertinente es privilegiar la información valiosa y relacionada con los temas propuestos inicialmente.

\section{El trabajo investigativo dentro de la dinámica institucional}

Más allá de la preocupación constante por diseñar propuestas que configuren proyectos realizables y pertinentes, se suma la necesidad de acomodar las apuestas investigativas a la lógica de trabajo propia de las instituciones de educación superior, donde primen en gran medida aspectos como la planeación, ejecución y muestra de resultados. En este sentido integrar lo institucional a los objetivos del quehacer investigativo puede ser visto con apatía y desgano pero -dadas nuestras experiencias- a la larga constituye una ventaja poder contar con herramientas que faciliten la comunicación y cooperación entre lo académico y lo institucional.

Dicha relación casi siempre marcada por fuertes sesgos tradicionalistas que consideran que la actividad intelectual está separada

ITINERARIO EDUCATIVO • AÑO XXVI, N. ${ }^{\circ} 59$ • I99-ZI4 • ENERO - JUNIO DE ZOIS 
de la maquinaria institucional, no toman en cuenta que las dos se complementan. También es cierto que en la práctica se pueden constatar varios momentos en que las políticas administrativas obstaculizan el tranquilo devenir del investigador. Por ejemplo, los apoyos económicos para asistir a eventos académicos como este, suelen estar precedidos por engorrosos trámites.

La angustia de los investigadores por sortear las mencionadas exigencias de las instituciones, dentro de todo presenta ventajas formativas, los estudiantes aprendemos a manejar presupuestos, solicitar materiales de papelería, realizar cronogramas, elaborar informes administrativos y actas de monitorias, entre otras.

A veces incluso tener presente las limitaciones presupuestales sirve para establecer los alcances reales y la necesidad de dividir grandes temas de preocupación académica en varios proyectos conectados entre sí. Nos atrevemos a decir que lo institucional contribuye en la formación de una especie de «malicia» investigativa.

\section{Elaboración y corrección de los resultados parciales y finales}

Jean Pierre Deslauriers (2005:106), afirma que «un informe que nunca se termina significa una investigación que permanece como proyecto, y en consecuencia cual quimera atormentará durante mucho tiempo la mente del investigador», no se trata de asimilar la investigación como una simple entrega de resultados -la concebimos como un proceso- pues sería una mirada reduccionista de nivel elemental, pero tener un concepción amplia no limita la efectividad del investigador y su conciencia acerca del deber de divulgar lo construido.

Los informes finales -resultados o académicos- constituyen el medio para hacer públicos los hallazgos $y$, por eso deben ocupar un puesto importante en la mente del indagador durante todas sus 
búsquedas. Quiere decir que, en cada ficha, lectura y entrevista el investigador deberá pensarse cómo puede ser mostrado cada resultado.

Se supone -y así lo creemos- que el informe también es el momento en que se explican las pesquisas encontradas, no olvidar la investigación como una búsqueda por diferentes caminos, no obstante palpar esos encuentros requiere de una labor de «artesanía intelectual», que Luis Jesús Galindo (1998), la refiere como una constante relación entre los objetivos formales desarrollados de manera tradicional y una alternativa apuesta por la creatividad investigativa.

En los informes finales que a los integrantes del semillero nos ha correspondido desarrollar, siempre hemos intentado relacionar los aspectos acabados de mencionar, no obstante, es difícil ser creativos -no imaginativo ni inventivos- cuando para ello se requiere de miradas panorámicas y oteadoras. Pero es muy confortante saber que para alcanzar el nivel de investigadores analíticos y metacognitivos, lo que más se requiere es continuar en el proceso de investigar y a la vez formarse.

Esa investigación pertinente para la formación, se da de varias maneras durante la elaboración de los resultados, pero una importante surge cuando el profesor y asesor corrige y explica cada una de las equivocaciones, justo ahí se produce la ruptura.

\section{La conciencia sobre el problema de las fuentes o el problema de los archivos}

El problema de las fuentes constituye un nuevo conocimiento pero más allá es el despertar de una conciencia sobre el estado de las fuentes en la Universidad y en la ciudad. Nos dimos cuenta que la preocupación por la conservación de los archivos y las hemerotecas en Pereira ha sido poca.

ITINERARIO EDUCATIVO • AÑO XXVI, N. ${ }^{\circ} 59$ • I99-ZI4 • ENERO - JUNIO DE ZOIL 
La manera como nos dimos cuenta de ello fue progresiva, inicialmente al irnos topando con la desaparición de archivos de años completos, o la observación de periódicos deteriorados por la humedad, rayados, con paginas arrancadas -las personas siempre arrancan los crucigramas pero no se dan cuenta que por detrás se llevan media noticia- que hacen incomprensible la información.

Otro dato curioso que surgió en esa constante indagación, tiene que ver con la ausencia de actas en el archivo de fechas específicas en donde según las entrevistas ocurrieron asuntos complejos importantes de resaltar. Coincidencia o no, complicado de demostrar lo uno o lo otro. Pero lo relevante es darse cuenta que para la investigación y más para la histórica la preservación de las fuentes es indispensable, y en esa medida la intención por salvaguardar los documentos históricos debería ser una constante de las instituciones, no es así.

Pero fulminante en este discernimiento resultó la visita realizada al Archivo Histórico de la Universidad Nacional, donde encontramos un sistema idóneo de manejo de la información histórica, sin obviar lo significativo de los fondos profesorales. En fin, parte de ese aprendizaje fue entender todas estas variables que se dan en torno a las fuentes que proporcionarán vida a la investigación.

\section{La importancia del docente-tutor en la aventura investigativa}

Durante los procesos investigativos, la adquisición de aprendizajes metodológicos y el acercamiento a las prácticas o actividades que constituyen los «saberes prácticos del joven investigador» -la elaboración de informes de lectura, interpretación de fuentes escritas y la elaboración de investigación mediante entrevistasvemos sobresalir la figura del docente tutor como participante y como observador. 
Nuestro tutor interviene durante las discusiones tratando de orientar las técnicas y teorías, delimitar el campo de acción de los estudiantes para lograr que nosotros no caigamos en la divagación conceptual -es complicado porque debe delimitarlos sin limitarlos- pero además es un observador sentado en la silla del director -pero al alcance del estudiante- husmeando, preguntando y tanteando sobre los conocimientos previos que cada uno de los estudiantes tenemos, articulando pasiones, necesidades, temores, frustraciones y cualidades. Toma el riesgo de invertir tiempo valioso que no le representa aparente continuidad en su proceso formativo para dedicarse a potenciar el debate, la discusión y la confrontación, aptitudes necesarias para dar rienda suelta a la «Artesanía Intelectual» que supone la fluctuación constante entre la reflexión y la práctica en torno a la problematización de la investigación.

\section{Conclusiones}

Todo lo plasmado y comentado en esta ponencia, hace parte de las experiencias de aprendizaje que han emergido desde el interés y los lineamientos del semillero del trabajo en equipo.

Han sido apuestas -arriesgadas- para obtener resultados satisfactorios, evidenciando en cada actividad el rigor académico. Las investigaciones ejecutadas por el semillero se han asumido con el compromiso y el trabajo de todos los días bajo la disciplina y dedicación que los investigadores consagrados invierten en sus proyectos. Por supuesto, no compartimos la postura que muestra a los semilleros como un grupo que se reúne esporádicamente para trabajar lecturas aisladas que suelen hacerse en las horas libres. Para nosotros el "aprender investigando» constituye la investigación y en esa medida la labor al interior del semillero es un quehacer que se construye y transforma a diario, donde fortalecer el compromiso es vital para lograr avances formativos.

ITINERARIO EDUCATIVO • AÑO XXVI, N. ${ }^{\circ} 59 \cdot 199-214 \cdot$ ENERO - JUNIO DE ZOIL 
Trabajar bajo los planteamientos de la investigación formativa es también romper con la clásica y conservadora manera de entender la investigación, donde el conocimiento ya está realizado y sólo hay que redescubrirlo y recordarlo. Después de que se transgreden los tradicionalismos el nuevo tipo de investigador -nos formamos en esa dirección-debe preocuparse en sus indagaciones por reflejar un diálogo entre la teoría y lo subjetivo -social y cultural- para lograr ser reflexivo y creativo. $\mathrm{Al}$ conseguirlo no perderá nada de lo que le sucede en su continuo aprendizaje.

\section{Referencias}

Delgado, A. (2011, octubre). Patrimonio Documental y Bibliográfico. Ponencia presentada en el Encuentro Regional de Semilleros, Tuluá-Valle del Cauca, Colombia.

Desliauriers, J. (2005). Investigación cualitativa, Guía práctica. Pereira: Papiro.

Galeano, E. (2004). Estrategias de investigación social cualitativa. El giro en la mirada. Medellín: La Carreta.

Galindo, C. (1998). Técnicas de investigación en sociedad, cultura y comunicación. México: Prentice Hall, Pearson Educación. Addison Wesley.

Gil, A. (2011, noviembre). Memorias Universitarias UTP. 50 años Marcando la Historia de la Región. Ponencia presentada en Encuentro de Estudiantes de Ciencias Sociales, Buga-Valle del Cauca, Colombia.

Létourneau, J. (2005). La caja de herramientas del joven investigador. Medellín: La Carreta. 